$$
\int_{C}\left|u(x)-u_{C}\right| d x \leqq K_{3} R^{n+1-n / p}\left(\int_{C}|\operatorname{grad} u(x)|^{p} d x\right)^{1 / p}
$$

and the desired result follows from the previous theorem.

This paper has been written with the support of the Office of Naval Research, under project Nonr 710 (16), NR-043-041.

\title{
REFERENCE
}

1. F. John and L. Nirenberg, On functions of bounded mean oscillation, Comm. Pure Appl. Math. 14 (1961), 415-426.

UnIVERSITY OF MiNNESOTA

\section{PHRAGMEN-LINDELÖF THEOREMS FOR SECOND ORDER QUASI-LINEAR ELLIPTIC PARTIAL DIFFERENTIAL EQUATIONS}

\author{
JOHN O. HERZOG ${ }^{1}$
}

Phragmén-Lindelöf theorems for uniformly elliptic partial differential equations have been the subject of several papers in recent years (see e.g. $[3 ; 4 ; 6 ; 7 ; 8 ; 10]$ ). Here we are concerned with the Phragmén-Lindelöf theorem for second order quasi-linear elliptic equations of the form

$$
L[z] \equiv \sum a_{i j}(x, p) z_{x_{i} x_{j}}=f(x, z, p),
$$

which need not be uniformly elliptic. The main result is Theorem 1 which roughly says that if $u(x)$ is a subfunction with respect to (1) in a domain $D$ contained in a half space and if $u(x) \leqq 0$ on the boundary of $D$ then either $u(x) \leqq 0$ throughout $D$ or the maximum of $u(x)$ on a sphere of radius $r$ is of order not less than $r^{\eta}$ for some $\eta>0$. Probably the most interesting feature of this theorem is that its proof essentially depends only on the behavior of the functions $a_{i j}(x, p)$ and $f(x, z, p)$ for $\sum p_{i}^{2} \leqq 1$. For $f \equiv 0$ and dimension $n=2$ it is shown that $\eta=1$.

Let $D$ be an unbounded domain contained in a half space of $n$ dimensional Euclidean space and let $T$ be the domain in $2 n$-dimen-

Received by the editors May 14, 1963.

1 This paper is a portion of a doctoral thesis written under the supervision of Professor Lloyd K. Jackson at the University of Nebraska. 
sional Euclidean space defined by $T \equiv\left\{(x, p) \equiv\left(x_{1}, \cdots, x_{n}, p_{1}, \cdots\right.\right.$, $\left.p_{n}\right): x \in D$ and $\left.-\infty<p_{1}, \cdots, p_{n}<+\infty\right\}$. Throughout the paper we shall use the notation: $p_{i}=\partial z / \partial x_{i}, r_{i j}=\partial^{2} z / \partial x_{i} \partial x_{j},\|x\|^{2}=\sum_{1}^{n} x_{i}^{2}$. $\sum$ will denote summation over one or both of the indices $i, j, 1 \leqq i$, $j \leqq n$.

Equation (1) is considered subject, at various times, to certain of the following conditions:

(i) The $a_{i j}(x, p)$ are continuous and have continuous first partial derivatives with respect to the $p_{i}$ for all $(x, p) \in T$.

(ii) $\sum a_{i j}(x, p) \mu_{i} \mu_{j}>0$ for all real $\mu_{1}, \cdots, \mu_{n}$ (not all zero) and all $(x, p) \in T$ (i.e. we assume pointwise ellipticity).

(iii) $a_{i j}=a_{j i}$ and the determinant of the $\left(a_{i j}\right)$ is identically one on $T$.

(iv) There exists an $\alpha_{0}>0$ such that $\left(\sum a_{i i}\right)^{n} \leqq \alpha_{0}$ for all $(x, p) \in T$ with $\sum p_{i}^{2} \leqq 1$.

(v) $f(x, z, p)$ is continuous and has continuous first partial derivatives with respect to $z, p_{1}, \cdots, p_{n}$, with $f_{z} \geqq 0$ for all $x \in D$ and for all $z$ and $p_{i}$.

(vi) There is a fixed $m>n^{1 / 2}$ such that there exist constants $\beta$ and $\gamma$ so that $|f(x, z, p)| \leqq \beta\left(p_{1}^{2}+\cdots+p_{n}^{2}\right)^{\gamma}$ for all $x \in D$ whenever $\sum p_{i}^{2} \leqq 1$, where $\gamma \geqq(2-\eta) /(2-2 \eta)$, and

$$
\eta=\log _{3} \frac{1-\exp \left[-8 m \alpha_{0} /(n-1)^{n-1}\right]}{1-\exp \left[-2 m \alpha_{0} /(n-1)^{n-1}\right]} .
$$

The proofs will be based on the following principle:

Maximum Principle [5]. Let $D$ be a bounded domain in Euclidean $n$-space and let $F\left(x_{1}, \cdots, x_{n}, z, p_{1}, \cdots, p_{n}, r_{11}, r_{12}, \cdots, r_{n n}\right)$ be continuous and have continuous first partial derivatives with respect to $z, p_{1}, \cdots, p_{n}, r_{11}, r_{12}, \cdots, r_{n-1, n}$ and $r_{n n}$ for all $x \in D$ and all $\left(z, p_{1}, \cdots, r_{n n}\right),-\infty<z, p_{1}, \cdots, p_{n}, r_{11}, r_{12}, \cdots, r_{n n}<\infty$. Furthermore, assume that $F_{z} \leqq 0$ and that the quadratic form $\sum F_{r_{i}} \mu_{i} \mu_{j}$ is positive definite for all $x \in D$ and all $\left(z, p_{1}, \cdots, r_{n n}\right)$. Then if $z_{1}(x)$ and $z_{2}(x) \in C^{(2)}(D)$, if the upper limit of $z_{1}(x)$ is less than or equal to the lower limit of $z_{2}(x)$ as $x$ approaches any boundary point of $D$, and if

$$
F\left(x_{1}, \cdots, x_{n}, z_{1}, z_{1 x_{1}} \cdots, z_{1 x_{n}}, z_{1 x_{1} x_{1}}, z_{1 x_{1} x_{2}}, \cdots, z_{1 x_{n} x_{n}}\right) \geqq 0
$$

in $D$ and

$$
F\left(x_{1}, \cdots, x_{n}, z_{2}, z_{2 x_{1}}, \cdots, z_{2 x_{n}}, z_{2 x_{1} x_{1}}, z_{2 x_{1} x_{2}}, \cdots, z_{2 x_{n} x_{n}}\right) \leqq 0
$$

in $D$, it follows that either $z_{1}<z_{2}$ in $D$ or $z_{1} \equiv z_{2}$ in $D$.

The conditions placed on equation (1) are such that $L[z]-f(x, z, p)$ satisfies the hypothesis placed on $F$ in the maximum principle. 
Theorem 1. Assume equation (1) satisfies conditions (i)-(vi). Let $u(x)$ satisfy in $D$ the inequality $L[u] \geqq f(x, u, p)$ and let the upper limit of $u(x)$ be nonpositive as $x$ approaches any point on the boundary of $D$. Then if

$$
\limsup _{r \rightarrow \infty} \frac{M(r)}{r^{\eta}} \leqq 0,
$$

where $M(r)=$ l.u.b. $u(x)$ for $\|x\|=r, x \in D$, it follows that $u(x) \leqq 0$ throughout $D$.

Proof. In addition to the previously defined notation we let

$$
\begin{aligned}
t^{2} & =x_{1}^{2}+\cdots+x_{n-1}^{2}, \quad G_{R}^{i}=D \cap\left\{x: t^{2}+\left(x_{n}+R\right)^{2} \leqq(i R)^{2}\right\}, \\
S_{R}^{i} & =\left\{x: t^{2}+\left(x_{n}+R\right)^{2}=(i R)^{2}, x_{n} \geqq 0\right\}, \\
N_{i}(R) & =\text { l.u.b. } u(x) \text { for } x \in S_{R}^{i} \cap D, \quad i=2,4 . \\
D_{i R} & =\{x: x \in D \text { and }\|x\| \leqq i R\}, \quad i=1,2,4 .
\end{aligned}
$$

Without loss of generality consider $D$ contained in the half space $x_{n}>0$. Assume $\lim \sup _{r \rightarrow \infty} M(r) / r^{n} \leqq 0$ and that $M\left(R^{\prime}\right)>0$ for some $R^{\prime}$ (and hence by the maximum principle for all $R \geqq R^{\prime}$ ). For $R \geqq R^{\prime}$ define

$$
w_{R}(x)=\frac{t^{2}+\left(x_{n}+R\right)^{2}}{4 R^{2}} .
$$

The following preliminary bounds will be used to show that there exists a constant $\alpha>0$, dependent on $\alpha_{0}, m$, and $n$, such that

$$
W_{R}(x) \equiv N_{2}(R) \frac{1-e^{-\alpha w_{R}}}{1-e^{-\alpha}}
$$

satisfies $L\left[W_{R}\right] \leqq f\left(x, W_{R}, W_{R x}\right)$ for all $x \in G_{R}^{4}$ when $R$ is sufficiently large. Note that by the maximum principle, $N_{2}(R)>0$.

By the Schwarz inequality,

$$
\left|\sum a_{i j} w_{R x_{i} x_{j}}\right| \leqq\left(\sum a_{i j}^{2}\right)^{1 / 2}\left(\sum w_{R x_{i} x_{j}}^{2}\right)^{1 / 2},
$$

and, since the quadratic form is assumed positive definite, we have $[2$, p. 32]

$$
\sum a_{i j} w_{x_{i}} w_{x_{j}} \geqq\left[\min _{1 \leqq i \leqq n} \lambda_{i}(x, p)\right] \sum w_{x_{i}}^{2},
$$

where the $\lambda_{i}(x, p)$ are the characteristic roots of $A \equiv\left(a_{i j}\right)$ at the point $(x, p) \in T$. Using a well-known inequality $[1$, p. 67], we have 


$$
\lambda_{\min }^{A} \geqq[\operatorname{det} A]\left[\frac{n-1}{\operatorname{Trace} A-\lambda_{\min }^{A}}\right]^{n-1} \geqq[\operatorname{det} A]\left[\frac{n-1}{\sum a_{i i}}\right]^{n-1},
$$

where $\lambda_{\min }^{A}$ denotes a minimum characteristic root of the real positive definite matrix $A$. Hence, since $\operatorname{det} A=1$,

$$
\sum a_{i j} w_{R x_{i}} w_{R x_{j}} \geqq\left[\frac{n-1}{\sum a_{i i}}\right]^{n-1} \sum w_{R x_{i}}^{2} .
$$

Also,

$$
\sum a_{i j}^{2}=\left(\sum a_{i i}\right)^{2}-\sum\left(a_{i i} a_{j j}-a_{i j}^{2}\right) \leqq\left(\sum a_{i i}\right)^{2} .
$$

Thus by (2), (3), and (4) above we see that

$$
\begin{aligned}
& L\left[W_{R}\right]-f\left(x, W_{R}, W_{R x}\right) \\
& =N_{2}(R) \frac{\alpha e^{-\alpha w_{R}}}{1-e^{-\alpha}}\left\{\sum a_{i j} w_{R x_{i} x_{j}}-\alpha \sum a_{i j} w_{R x_{i}} w_{R x_{j}}\right\}-f\left(x, W_{R}, W_{R x}\right) \\
& \text { (5) } \\
& \leqq \frac{(n-1)^{n-1} N_{2}(R) \alpha e^{-\alpha w R} \sum w_{R x_{i}}^{2}}{\left(1-e^{-\alpha}\right)\left(\sum a_{i i}\right)^{n-1}} \\
& \cdot\left\{\frac{\left(\sum a_{i i}\right)^{n}\left[\sum w_{R x_{i} x_{j}}^{2}\right]^{1 / 2}}{(n-1)^{n-1} \sum_{w_{R x_{i}}^{2}}^{2}}-\alpha-\frac{\left(1-e^{-\alpha}\right)\left(\sum a_{i i}\right)^{n-1} f\left(x, W_{R}, W_{R x}\right)}{N_{2}(R) \alpha(n-1)^{n-1} e^{-\alpha w_{R}} \sum w_{R x_{i}}^{2}}\right\} \\
& \equiv G \cdot H,
\end{aligned}
$$

where $H$ denotes the expression contained in the braces.

Now,

$$
1 / 4 R^{2} \leqq \sum w_{R x_{i}}^{2} \leqq 4 / R^{2} \quad \text { for } x \in G_{R}^{4}
$$

and $w_{R x_{i} x_{i}}=1 / 2 R^{2}$ while $w_{R x_{i} x_{j}}=0$ for $i \neq j$. Therefore

(7) $\left(\sum w_{R x_{i} x_{j}}^{2}\right)^{1 / 2} / \sum w_{R x_{i}}^{2} \leqq\left[\left(n / 4 R^{4}\right)^{1 / 2}\right] /\left[1 / 4 R^{2}\right]=2 n^{1 / 2}$ for $x \in G_{R}^{4}$.

Since $w_{R}(x) \geqq 1 / 4$ for $x \in D$ (independent of $R$ ),

$$
\left[4 \alpha^{2} e^{-2 \alpha w_{R}(x)}\right] /\left(1-e^{-\alpha}\right)^{2}
$$

is bounded (independent of $R$ and $\alpha>0$ ) by a constant $K_{1}$ for all $x \in D$. Thus

$$
\begin{aligned}
\sum W_{R x_{i}}^{2}(x) & =N_{2}^{2}(R) \frac{\alpha^{2} e^{-2 \alpha w_{R}(x)}}{\left(1-e^{-\alpha}\right)^{2}} \sum w_{R x_{i}}^{2} \\
& \leqq K_{1} N_{2}^{2}(R) / R^{2} \quad \text { for all } x \in G_{R}^{4} .
\end{aligned}
$$


It follows from hypothesis (iii) that $\prod_{1}^{n} \lambda_{i}=1$. But by the theorem on the geometrical and arithmetical mean,

$$
\left(\sum \lambda_{i}\right) / n \geqq\left(\prod_{1}^{n} \lambda_{i}\right)^{1 / n}=1
$$

Therefore $\alpha_{0} \geqq\left(\sum a_{i i}\right)^{n}=\left(\sum \lambda_{i}\right)^{n} \geqq n^{n}$. Thus for any fixed $m>n^{1 / 2}$ we have $m \alpha_{0} /(n-1)^{n-1}>n \sqrt{ } n \geqq 2 \sqrt{ } 2$ and we see from the expression for $\eta$ that $0<\eta<1$. Hence $\lim \sup _{R \rightarrow \infty} M(R) / R^{\eta} \leqq 0$ implies $\lim \sup _{R \rightarrow \infty} M(2 R) / R \leqq 0$ and since $D_{2 R} \supset G_{R}^{2}$ we can choose $R^{*}>R^{\prime}$ sufficiently large so that for $R \geqq R^{*}, N_{2}^{2}(R) / R^{2} \leqq 1 / K_{1}$. Thus $\sum W_{R x_{i}}^{2}(x)$ $\leqq 1$ for all $x \in G_{R}^{4}, R \geqq R^{*}$. Consequently, by hypotheses (iv) and (vi), respectively,

and

$$
\left[\sum a_{i i}\left(x, W_{R x}\right)\right]^{n} \leqq \alpha_{0}
$$

$$
\left|f\left(x, W_{R}, W_{R x}\right)\right| \leqq \beta\left(\sum W_{R x_{i}}^{2}\right)^{\gamma} \quad \text { for all } x \in G_{R}^{4} .
$$

Expression $G$ of (5) is positive and by (7), (8), and (9) $H$ is bounded by

$$
\begin{aligned}
\frac{2 n^{1 / 2} \alpha_{0}}{(n-1)^{n-1}}-\alpha & \\
+\frac{\left(\sum a_{i i}\right)^{n-1} \beta \alpha^{2 \gamma-1} e^{-\alpha(2 \gamma-1) w_{R}(x)}\left(\sum w_{R x_{i}}^{2}\right)^{\gamma-1} N_{2}^{2 \gamma-1}(R)}{(n-1)^{n-1}\left(1-e^{-\alpha}\right)^{2 \gamma-1}} & \text { for all } x \in G_{R}^{4} .
\end{aligned}
$$

Since $\gamma \geqq(2-\eta) /(2-2 \eta)>1$ and $w_{R}(x) \geqq 1 / 4$ in $G_{R}^{4}$ we have that

$$
\frac{\alpha^{2 \gamma-1} e^{-\alpha(2 \gamma-1) w_{R}(x)}}{\left(1-e^{-\alpha}\right)^{2 \gamma-1}} \leqq J,
$$

where $J$ is a constant independent of $R$ and $\alpha>0$. Therefore it follows from (6) and hypothesis (iv) that (10) is

$$
\leqq \frac{2 n^{1 / 2} \alpha_{0}}{(n-1)^{n-1}}-\alpha+\frac{4^{\gamma-1} \alpha_{0}^{(n-1) / n} \beta J N_{2}^{2 \gamma-1}(R)}{(n-1)^{n-1} R^{2 \gamma-2}} .
$$

Because lim $\sup _{R \rightarrow \infty} M(R) / R^{\eta} \leqq 0$ we may choose $R^{* *}>R^{*}$ such that

$$
\frac{N_{2}^{2 \gamma-1}(R)}{R^{2 \gamma-2}} \leqq\left[\frac{N_{2}(R)}{R^{\eta}}\right]^{2 \gamma-1}<\frac{2 \alpha_{0}\left[m-n^{1 / 2}\right]}{4^{\gamma-1} \alpha_{0}^{(n-1) / n} \beta J}
$$


for all $R \geqq R^{* *}$. Thus, retracting the preceding inequalities, we see that $\alpha=2 m \alpha_{0} /(n-1)^{n-1}$ suffices to make

$$
L\left[W_{R}\right]-f\left(x, W_{R}, W_{R x}\right) \leqq 0 \quad \text { for all } x \in G_{R}^{4}, R \geqq R^{* *} .
$$

Throughout the remainder of this proof we consider an arbitrary but fixed $R>R^{* *}$. Assume $u(x) \leqq W_{R}(x)$ for all $x \in D \cap S_{R}^{*}$. Now $u\left(x_{0}\right)=N_{2}(R)$ for some point $x_{0} \in D \cap S_{R}^{2}, x_{0}$ interior to $G_{R}^{4}$, and $W_{R}(x)=N_{2}(R)$ on $S_{R}^{2}$. Therefore it follows from the maximum principle that $W_{R}(x) \equiv u(x)$ in $G_{R}^{4}$ However, $u(x)<W_{R}(x)$ at some points of $D \cap S_{R}^{2} \subset G_{R}^{4}$. Consequently $u(x)>W_{R}(x)$ at some points of $D \cap S_{R}^{4}$, so

$$
N_{4}(R) \geqq \min _{D \cap S_{R}^{4}} W_{R}(x)=N_{2}(R) \frac{1-e^{-4 \alpha}}{1-e^{-\alpha}},
$$

and hence, for any positive integer $j$,

$$
N_{4}\left(3^{j} R\right) \geqq N_{2}\left(3^{j} R\right) \frac{1-e^{-4 \alpha}}{1-e^{-\alpha}} .
$$

Now for every $R, G_{R}^{4} \subset G_{3 R}^{2}, G_{R}^{4} \subset D_{4 R}$, and $D_{R} \subset G_{R}^{2}$, so it follows by the maximum principle again that $N_{2}\left(3^{i} R\right) \geqq N_{4}\left(3^{j-1} R\right), M\left(4 \cdot 3^{i} R\right)$ $\geqq M_{4}\left(3^{i} R\right)$, and $N_{2}(R) \geqq M(R)$. Hence by iteration we have

$$
M\left(4 \cdot 3^{j} R\right) \geqq M(R) \sigma^{j+1} \quad \text { where } \quad \sigma=\frac{1-e^{-4 \alpha}}{1-e^{-\alpha}} .
$$

Denoting $4 \cdot 3^{i} R$ by $R_{j}$ we have

$$
M\left(R_{j}\right) \geqq M(R) \sigma^{j+1}=K^{\prime} R_{j}^{\log _{3} \sigma}, \quad K^{\prime}=\frac{M(R) \sigma}{(4 R)^{\log _{3} \sigma}},
$$

from which we conclude that $\lim _{\inf _{R \rightarrow \infty}} M(R) / R^{\eta}>0$. Thus we have a contradiction and we conclude that $u(x) \leqq 0$ in $D$.

Theorem 2. Assume that (1) satisfies conditions (i), (ii), and (iii) with $f \equiv 0$ and that $D$ is contained in the $n$-dimensional "cone" $x_{n} \geqq r \sin \alpha$ for some $\alpha, 0<\alpha<\pi / 2$. Let $u(x)$ satisfy in $D$ the inequality $L[u] \geqq 0$ and let the upper limit of $u(x)$ be nonpositive as $x$ approaches any point on the boundary of $D$. Then if

$$
\limsup _{r \rightarrow \infty} \frac{M(r)}{r} \leqq 0
$$

it follows that $u(x) \leqq 0$ throughout $D$. 
Proof. Assume that $\lim \sup _{r \rightarrow \infty} M(r) / r \leqq 0$ and that $M\left(R_{1}\right)>0$ for some $R_{1}$. By continuity of $u, u\left(x_{0}\right)=M\left(R_{1}\right)$ for some $x_{0}$ on $D \cap\{x:\|x\|$ $\left.=R_{1}\right\}$. For $0<k_{0}<M\left(R_{1}\right) / R_{1}$ we see that $z(x) \equiv k_{0} x_{n}<M\left(R_{1}\right)$ on $\|x\|=R_{1}, x \in D$; hence $z\left(x_{0}\right)<u\left(x_{0}\right)$. Since lim $\sup _{R \rightarrow \infty} M(R) / R \leqq 0$ we may, for $0<\epsilon<k_{0} \sin \alpha$, choose $R_{\epsilon}>R_{1}$ such that $M\left(R_{\epsilon}\right)<\epsilon R_{\epsilon}$. Therefore, for $\|x\|=R_{\epsilon}, x \in D$, we have

$$
u(x) \leqq \epsilon R_{\epsilon} \leqq k_{0} R_{\epsilon} \sin \alpha \leqq z(x) .
$$

Hence by the maximum principle $u(x) \leqq z(x)$ in $D \cap\left\{x:\|x\| \leqq R_{\varepsilon}\right\}$, a contradiction. It follows that $u(x) \leqq 0$ in $D$.

Theorem 3. For dimension $n=2$, the preceding theorem holds for any domain $D$ contained in a half plane.

Proof. It suffices to consider $D$ contained in the half plane $y>0$. For any $\epsilon>0$ define

and let

$$
u_{\epsilon}(x, y)=-\epsilon y+u(x, y)
$$

$L_{\epsilon}[z]=a(x, y, p, q+\epsilon) z_{x x}+2 b(x, y, p, q+\epsilon) z_{x y}+c(x, y, p, q+\epsilon) z_{y y}$, where $p=z_{x}, q=z_{y}$.

Choose $R_{\epsilon}>0$ such that $u(x, y) \leqq(\epsilon / 2) R$ for $x^{2}+y^{2}=R^{2} \geqq R_{\epsilon}^{2}$. Then for $y \geqq R_{\mathrm{e}}$ we will have

$$
u_{\epsilon}(x, y) \leqq-\epsilon R_{\epsilon}+(\epsilon / 2) R_{\epsilon}<0 .
$$

Assume $u_{\epsilon}(0, y)>0$ for some $y^{\prime}, 0<y^{\prime}<R_{\epsilon}$. Then $u_{\epsilon}(0, y)$ attains a positive maximum $M_{\mathrm{e}}$ at some point $\left(0, y_{0}\right), 0<y_{0}<R_{\epsilon}$. Define

$$
v_{\epsilon}(x, y)=u_{\epsilon}(x, y)-M_{\epsilon} .
$$

We may apply Theorem 2 to $L_{\epsilon}[z]=0$ and $v_{\epsilon}$ in $D_{1} \equiv D \cap\{$ first quadrant $\}$ and $D_{2} \equiv D \cap\{$ second quadrant $\}$ individually to conclude that $u_{\epsilon}(x, y) \leqq M_{\epsilon}$ throughout $D$.

Next consider $D_{R_{1}} \equiv D \cap\left\{(x, y): x^{2}+y^{2} \leqq R_{1}^{2}\right\}$ for any $R_{1}>R_{\epsilon}$. At the point $\left(0, y_{0}\right)$ interior to $D_{R_{1}}$ we have $u_{\epsilon}\left(0, y_{0}\right)=M_{\epsilon}$; hence by the maximum principle $u_{\epsilon} \equiv M_{\epsilon}$ in $D_{R_{1}}$, which is a contradiction since $u_{\epsilon}\left(0, R_{\epsilon}\right) \leqq 0<M_{\epsilon}$. Therefore $u_{\epsilon}(0, y) \leqq 0$ for all $y \geqq 0,(0, y) \in D$. Now apply Theorem 2 to $L_{\epsilon}[z]=0$ and $u_{\epsilon}$ in $D_{1}$ and $D_{2}$ individually to conclude that $u(x, y) \leqq \epsilon y$ throughout $D$. Since $\epsilon$ is arbitrary we conclude that $u(x, y) \leqq 0$ throughout $D$.

\section{REFERENCES}

1. E. Bodewig, Matrix calculus, Interscience, New York, 1956.

2. R. Courant and D. Hilbert, Methods of mathematical physics, Vol. 1, Interscience, New York, 1953. 
3. Avner Friedman, On two theorems of Phragmen-Lindelöf for linear elliptic and parabolic differential equations of the second order, Pacific J. Math. 7 (1957), 1563-1575.

4. David Gilbarg, The Phragmen-Lindelöf theorem for elliptic partial differential equations, J. Rational Mech. Anal. 1 (1952), 411-417.

5. E. Hopf, Elementarie Betrachtungen über die Losungen partieller Differentialgleichungen zweiter Ordnung vom elliptischen Typus, S.-B. Preuss. Akad. Wiss. 19 (1927), 147-152.

6. - Remarks on the preceding paper by D. Gilbarg, J. Rational Mech. Anal. 1 (1952), 419-424.

7. E. M. Landis, Some questions on the qualitative theory of elliptic and parabolic equations, Uspehi Mat. Nauk 14 (1959), 22-85; Amer. Math. Soc. Transl. (2) 20 (1962), 173-238.

8. - Some questions of the qualitative theory of elliptic equations of the second order, Uspehi Mat. Nauk 18 (1963), 3-62.

9. E. Phragmén and E. Lindelöf, Sur une extension d'un principe classique de l'analyse, Acta Math. 31 (1908), 381-406.

10. J. B. Serrin, On the Phragmen-Lindelöf principle for elliptic differential equations, J. Rational Mech. Anal. 3 (1954), 395-413.

UNIVERSITY OF NEBRASKA 